\title{
Quality of Care for Patients with Multiple Chronic Conditions: The Role of Comorbidity Interrelatedness
}

\author{
Donna M. Zulman, MD, MS ${ }^{1,2}$, Steven M. Asch, MD, MPH ${ }^{1,2}$, Susana B. Martins, MD, MSc ${ }^{3}$, \\ Eve A. Kerr, $M D, M P H^{4,5}$, Brian B. Hoffman, $M D^{6,7}$, and Mary K. Goldstein, $M D, M S^{3,8}$
}

${ }^{1}$ Center for Health Care Evaluation, VA Palo Alto Health Care System, Menlo Park, CA, USA; '2Division of General Medical Disciplines, Department of Internal Medicine, Stanford University, Stanford, CA, USA; ${ }^{3}$ Geriatrics Research Education and Clinical Center, VA Palo Alto Health Care System, Palo Alto, CA, USA; ${ }^{4}$ Center for Clinical Management Research, VA Ann Arbor Healthcare System, Ann Arbor, MI, USA; ${ }^{5}$ Department of Internal Medicine, University of Michigan, Ann Arbor, MI, USA; ${ }^{6}$ VA Boston Health Care System, West Roxbury Division, Boston, MA, USA; ${ }^{7} \mathrm{Harvard}$ Medical School, Boston, MA, USA; ${ }^{8}$ Center for Primary Care and Outcomes Research, Stanford University, Stanford, CA, USA.

Multimorbidity - the presence of multiple chronic conditions in a patient-has a profound impact on health, health care utilization, and associated costs. Definitions of multimorbidity in clinical care and research have evolved over time, initially focusing on a patient's number of comorbidities and the associated magnitude of required care processes, and later recognizing the potential influence of comorbidity characteristics on patient care and outcomes. In this article, we review the relationship between multimorbidity and quality of care, and discuss how this relationship may be mediated by the degree to which conditions interact with one another to generate clinical complexity (comorbidity interrelatedness). Drawing on established theoretical frameworks from cognitive engineering and biomedical informatics, we describe how interactions among conditions result in clinical complexity and may affect quality of care. We discuss how this comorbidity interrelatedness influences the value of existing quality guidelines and performance metrics, and describe opportunities to quantify this construct using data widely available through electronic health records. Incorporating comorbidity interrelatedness into conceptualizations of multimorbidity has the potential to enhance clinical and research efforts that aim to improve care for patients with multiple chronic conditions.

J Gen Intern Med 29(3):529-37

DOI: $10.1007 / \mathrm{s} 11606-013-2616-9$

(c) Society of General Internal Medicine 2013

$\mathrm{M}$ ultimorbidity - the presence of multiple chronic conditions - is an increasingly recognized challenge to quality improvement and cost-containment efforts for healthcare systems. When patients have multiple health problems, it affects their quality of life and ability to work,

Received April 29, 2013

Revised July 30, 2013

Accepted August 26, 2013

Published online October 1, 2013 and is associated with increased rates of functional decline and mortality. ${ }^{1-5}$ Furthermore, as a patient's number of chronic conditions increases, there is a rise in associated physician visits, prescriptions, hospitalizations, home health visits, out-of-pocket expenses, and total health care expenditures. ${ }^{1,6-9}$

In order to optimize interventions and policies addressing multimorbidity, we must have a clear understanding of the relationship between multimorbidity and quality of care. In this paper, we describe how a patient's number of chronic conditions and the characteristics of those conditions have been observed to affect quality of care. We highlight comorbidity interrelatedness - the degree to which conditions interact to affect clinical management-as an important dimension of multimorbidity that presents additional quality-of-care challenges. We propose that adoption of a multimorbidity framework that integrates a patient's number of conditions, the characteristics of those conditions, and their interrelatedness, is likely to inform quality improvement efforts to enhance care for patients with multiple chronic conditions.

\section{REVIEW OF MULTIMORBIDITY AND ITS RELATIONSHIP WITH QUALITY OF CARE \\ Definition of Multimorbidity}

Among the different taxonomies of multimorbidity that have been proposed, ${ }^{10-14}$ a representative and widely used definition is the coexistence of two or more chronic conditions, where one is not necessarily more central than others. ${ }^{15}$ In research and quality improvement efforts, multimorbidity is often reduced to a count of conditions, particularly when investigators desire a simple analytic tool to adjust for variation in comorbidity number. ${ }^{16-18}$ It is generally accepted, however, that multimorbidity measures should-when feasible - consider disease severity and the burden of physiological dysfunction resulting from multiple conditions and 
affected systems. ${ }^{18,19}$ Several multimorbidity indices, including the Charlson score ${ }^{20,21}$ and the Index of Coexisting Disease ${ }^{22}$ incorporate disease severity. Although these indices tend to underrepresent some conditions, they have been shown to reliably predict outcomes such as disability and mortality, ${ }^{18}$ and can be of value when controlling for differences across patient populations. ${ }^{23}$

Multimorbidity can be challenging to conceptualize because of the heterogeneity intrinsic to chronic conditions, including variability in terms of symptom burden, risk of adverse clinical outcomes, self-management requirements, and the need for a specialist's care. This heterogeneity is intensified by variation in the degree to which conditions are related to one another. For example, some conditions are considered related or concordant in terms of their pathogenesis (e.g., hypertension, heart disease, and hyperlipidemia), while others are considered unrelated or discordant (e.g., heart disease, depression, and asthma), leading to very different management needs and treatment strategies. ${ }^{24,25}$ As a result, clinically meaningful evaluations of multimorbidity need to consider the specific qualities of individual conditions, as well the qualities of common condition dyads and triads. ${ }^{9,26,27}$

While multimorbidity generally refers to the presence of multiple clinical conditions, there is also growing recognition that a multitude of patient-level factors independent of specific comorbid conditions may complicate care and affect outcomes for multimorbid patients. ${ }^{10,28,29}$ For example, Safford et al. proposed a conceptual approach to complex patients that involves interactions between biological, socioeconomic, cultural, environmental, and behavioral forces as health determinants. ${ }^{10}$ Incorporating these dimensions into clinical assessments is likely to help ensure that care is aligned with patient's preferences, goals, and needs. ${ }^{30}$

\section{Clinical Challenges Generated by Multimorbidity}

Clinicians, especially those practicing in primary care, geriatrics, and mental health care, frequently encounter a number of challenges in caring for patients with multiple chronic conditions. ${ }^{30}$ Patients often present with a large number of medical issues (and accompanying medications), and these must be addressed in a brief clinic visit. Some of the conditions may be clinically dominant (e.g., end-stage renal disease) or highly symptomatic (e.g., chronic pain), thereby consuming a substantial amount of a clinician's time and drawing attention away from other health problems. $^{24,31}$ Furthermore, when two conditions are discordant in terms of their pathogenesis (e.g., diabetes and depression), they may require separate, time-intensive treatment plans. ${ }^{24}$ Valderas et al. describe how conditions may interact in other ways that can have either positive or negative consequences. For example, diagnosis of age- related macular retinopathy may occur earlier in patients with diabetes because they undergo regular fundus examinations. In these same patients, however, coronary heart disease may go undetected due to their altered pain sensation. ${ }^{12}$ These interactions generate a need for greater vigilance when evaluating patients with comorbid conditions. In addition, challenges frequently arise because of the shortage of clinical trial evidence describing interactions among conditions and treatments in patients with multimorbidity, as noted by the American Geriatrics Society in its guidelines for caring for older adults with multiple chronic conditions. ${ }^{30}$ Consequently, there is a great need for practical guidance and tools to support clinicians' management of multimorbid patients.

\section{The Relationship Between Multimorbidity and Quality of Care}

The relationship between multimorbidity and quality of care has been examined extensively in recent years. While some studies have found that higher comorbidity burden is associated with better quality of care, ${ }^{32,33}$ this relationship is inconsistent ${ }^{34}$ and appears to vary substantially depending on a patient's specific conditions and combinations of conditions. ${ }^{35}$ Several studies have documented quality of care deficiencies when patients have discordant or unrelated comorbidities. For example, Turner et al. found that hypertension management was less aggressive if a patient had unrelated comorbidities such as arthritis, cancer, or depression. ${ }^{36}$ Others have determined that patients with mental health conditions or chronic pain are at risk for receiving suboptimal care for their hypertension, diabetes, heart failure, arthritis, and chronic obstructive pulmonary disease. $^{31,36-40}$ Other studies, however, suggest that these patterns are not universal. For example, patients with serious mental health conditions sometimes receive more intensive monitoring of their other chronic conditions. ${ }^{41}$

There are several factors that may help reconcile the conflicting observations about multimorbidity and quality of care. Multimorbid patients tend to have frequent and intensive contact with the health care system, ${ }^{1,9}$ resulting in more opportunities to monitor each of their conditions, adjust their treatment regimens, and assess their general health maintenance needs. ${ }^{32,41}$ At the same time, each additional condition generates opportunities for suboptimal management, including missed diagnoses, inadequate treatment, and access and communication barriers. If a patient has conditions that are unrelated or discordant, there may be insufficient time or competing demands during a visit, ${ }^{31,37,42}$ and the patient and his or her provider may disagree about which condition should be prioritized. ${ }^{42,43}$ Additional challenges arise when patients have several clinicians involved in their care, increasing the likelihood of conflicting advice, redundant diagnostic tests and 
services, and impractical treatment regimens. ${ }^{1,44}$ Finally, it should be noted that in some instances, traditional quality measures may not represent appropriate goals for patients with multiple chronic conditions. ${ }^{44}$ Few quality measures account for these circumstances, however, making it difficult to interpret aggregate quality scores for heterogeneous populations.

Quality of care for multimorbid patients is therefore likely influenced by a patient's number of conditions, as well as by characteristics of specific conditions that reduce the amount of time or attention that are available for other issues (as may occur when conditions are symptomatic or clinically dominant). An additional influential factor - one that is appreciated by many clinicians but has not yet been integrated into quality of care metrics and tools - is comorbidity interrelatedness, or the degree to which conditions interact with one another in ways that generate clinical complexity.

In the following sections, we describe comorbidity interrelatedness and its relationship to quality of care in greater detail, and discuss opportunities to incorporate it into clinical guidelines, performance measures, and research.

\section{INTEGRATING COMORBIDITY INTERRELATEDNESS INTO A QUALITY OF CARE FRAMEWORK FOR MULTIMORBIDITY}

\section{Comorbidity Interrelatedness: A Source of Complexity in Multimorbid Patients}

In order to understand the role of comorbidity interrelatedness in care for multimorbid patients, it is helpful to draw on established models of complex systems, ${ }^{45}$ including frameworks that have been developed within the fields of cognitive engineering ${ }^{46,47}$ and biomedical informatics. ${ }^{48}$ According to these models, the complexity of any given system or task is influenced by the number of components comprising the system or task (i.e., component complexity), and the number and qualities of relationships among the components (i.e., coordinative complexity). ${ }^{46,48}$ Within the context of health care, these concepts have been used to evaluate complex clinical decisions and healthcare system work flow. ${ }^{47-50}$

By conceptualizing patients with multiple chronic conditions as complex systems, one can begin to explore and potentially quantify the contributions of component complexity (i.e., number and characteristics of an individual's conditions) and coordinative complexity (i.e., comorbidity interrelatedness) to quality of care (Fig. 1). In this context, comorbidity interrelatedness represents the ways in which comorbid conditions interact with one another to generate clinical complexity. Such interactions may lead to diagnostic uncertainty, or may influence choice of medications or the relative risks or benefits of treatment. Of note, these interactions can occur in the context of both concordant and discordant conditions, because in either scenario, the presence of one condition may influence clinical management of the other. Table 1 provides examples of how comorbidity interrelatedness may influence the quality of care for specific processes such as screening, diagnosis, treatment, and follow-up.

To illustrate these concepts, consider how comorbid conditions influence hypertension management decisions. While selection of an anti-hypertensive medication may be relatively straightforward when a patient has no comorbid conditions, the decision process becomes more complicated when a patient has multiple chronic conditions, because of the potential benefits and risks associated with comorbidity-medication interactions. For example, thiazide medications may be harmful in a patient with gout because they increase hyperuricemia and can lead to a gout flare, but the same medications may be beneficial in a patient with osteoporosis because they decrease calcium excretion and can increase bone mineral density. Both gout and osteoporosis therefore contribute to the clinical complexity of hypertension management, because they influence decisions about optimal medications. This complexity is compounded in patients with both conditions, because of the need to balance the potential benefits and risks of treatment with a thiazide. As Figure 2 illustrates, the clinical complexity generated by this type of comorbidity interrelatedness depends on the specific conditions that are present, and may be quite variable even among patients with similar numbers of conditions.

Importantly, comorbidity interrelatedness does not account for the full range of biological, socioeconomic, cultural, environmental, and behavioral forces that contribute to a patient's health and overall complexity (Fig. 1). ${ }^{10,12,29}$ These dimensions may moderate the relationship between comorbidity interrelatedness and quality of care, especially if certain characteristics play a dominant role in management decisions (e.g., if a patient prefers not to escalate treatment) or characteristics interact with conditions in ways that shape treatment decisions (e.g., if a patient's history of medication adherence or level of social support favors one treatment modality over another). These relationships highlight opportunities to enhance quality of care for complex patients with multiple chronic conditions; for example, by ensuring that they have ample time to discuss their health challenges and competing demands with their providers, and by offering services from social work, mental health providers, and other support staff.

\section{Comorbidity Interrelatedness and Quality of Care}

There are a number of ways in which interactions among conditions may generate clinical complexity and impede the delivery of high-quality, guideline-concordant care to multimorbid patients (Fig. 1). Comorbidity interrelatedness 


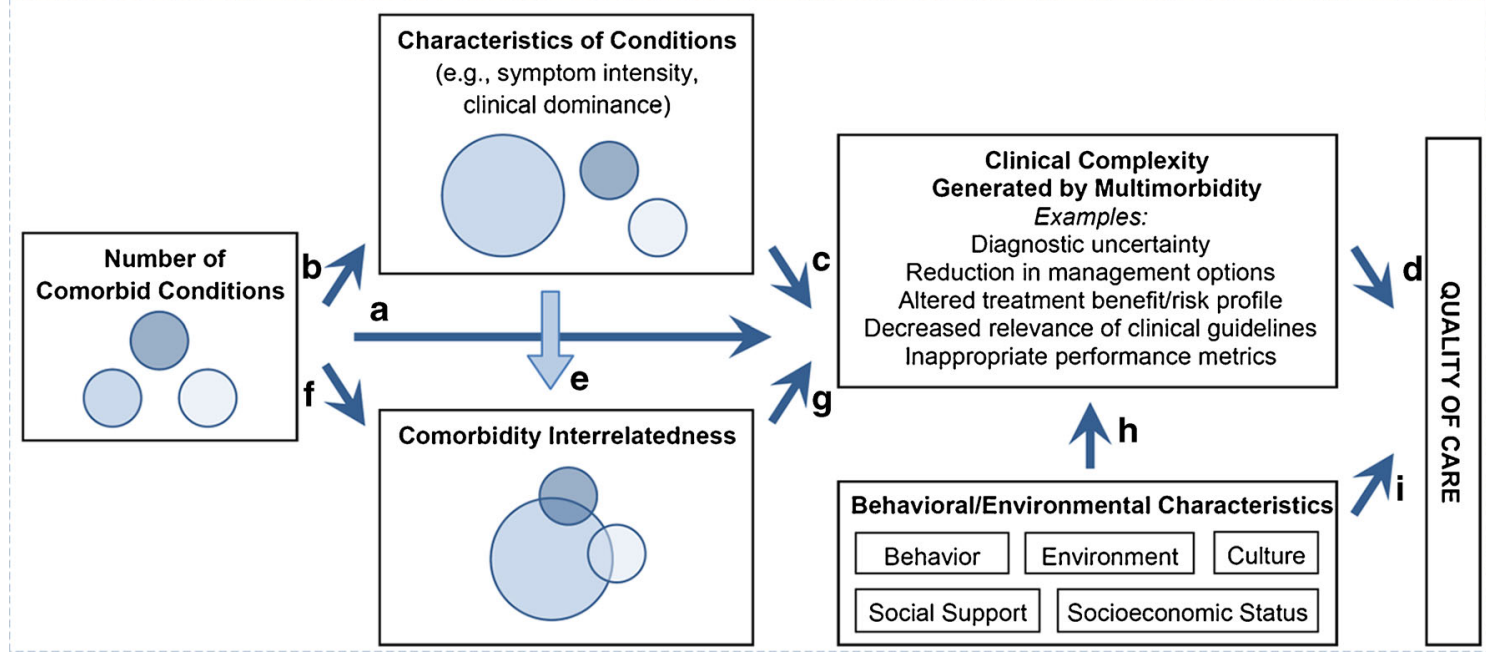

Figure 1. Conceptual framework depicting the influence of comorbidity number, characteristics, and interrelatedness on clinical complexity and quality of care for patients. In the proposed framework, the relationship between multimorbidity and quality of care is influenced by a patient's number of chronic conditions, comorbidity characteristics (e.g., symptom intensity, clinical dominance) and comorbidity interrelatedness, all of which increase clinical complexity. A patient's sheer number of conditions can generate clinical complexity because of the cognitive burden required to consider multiple clinical issues at once (a). With an increase in number of conditions, there is an increased

likelihood that one or more conditions may be symptomatic or dominant due to clinical severity (b), all of which may increase clinical complexity (c) and generate quality of care challenges (d). These condition characteristics also increase the likelihood of conditions interacting with one another in ways that affect management decisions, thereby increasing comorbidity interrelatedness (e). Comorbidity interrelatedness frequently occurs when two conditions are discordant or unrelated in their pathogenesis, but can also occur in the presence of conditions that are asymptomatic and concordant (e.g., diabetes influences the medication of choice for hypertension) (f). Comorbidity interrelatedness can increase clinical complexity through a number of mechanisms, as depicted in the box entitled, "Clinical complexity generated by multimorbidity" (g). Outside of the clinical domain, there are a number of important contextual factors, such as a patient's behavior, social support, and environment, that can also generate clinical complexity (h) and quality of care challenges (i), and can moderate the relationship between comorbidity interrelatedness and quality of care.

can influence decisions about optimal treatment, as occurs when the benefits and risks of treatment for one condition are affected by the presence of other conditions. ${ }^{51}$ For example, among patients with cancer, individuals with comorbid conditions frequently derive less benefit or greater harm from cancer treatment. ${ }^{52}$ Similarly, studies have found that as many as one-third of patients with diabetes have comorbid conditions that decrease the benefits and/or increase the risks of glycemic control. ${ }^{53}$

In addition, when patients have multiple conditions, their providers need to consider the clinical guidelines for each of the patient's individual conditions. The resulting list of guidelines can be long and unwieldy, and - if followed - can lead to impractical and potentially harmful care. ${ }^{15}$ The problem is not only the sheer number of recommended practices, but the fact that adhering to a guideline for one condition may exacerbate a second condition or increase risks associated with that disease. ${ }^{51}$ Boyd et al. illustrated this scenario memorably by describing the recommended guidelines for a hypothetical older patient with five common chronic conditions (osteoporosis, osteoarthritis, diabetes, hypertension, and chronic obstructive pulmonary disease). In order for a treatment plan to be adherent to all relevant guidelines, the patient's regimen would need to include 12 medications and 14 non-pharmacologic therapies, setting the stage for several possible drug-condition, drug-drug, and food-drug interactions. ${ }^{44}$

Qualitative studies with providers have exposed additional challenges generated by interrelated comorbidities. ${ }^{54}$ In one study in the U.K., providers noted that problems arise when clinical options for managing one condition are limited by the presence of another condition, ${ }^{55}$ as occurs when a patient would benefit from cardiovascular exercise, but is limited in the type of exercise due to osteoarthritis or obstructive pulmonary disease, or when dietary recommendations for two conditions conflict (as occurs with protein recommendations for diabetes and gout). Providers also express that interrelated conditions can impede or complicate the diagnostic process. For example, if a patient presents with abdominal pain and has a history of intestinal ischemia and irritable bowel syndrome, the differential includes both of these conditions, in addition to other causes. ${ }^{56}$ Of note, patients have voiced similar challenges in their self-care. For example, patients with heart failure report that it can be difficult to determine whether their symptoms are arising from their heart failure or from their diabetes or chronic obstructive pulmonary disease, which 
Table 1. Examples of Quality of Care Challenges Generated by Comorbidity Interrelatedness

\begin{tabular}{|c|c|c|}
\hline Care function* & $\begin{array}{l}\text { Quality of care challenges generated by comorbidity } \\
\text { interrelatedness }\end{array}$ & Examples \\
\hline Screening & $\begin{array}{l}\text { Screening recommendations for Condition A may } \\
\text { not be relevant if a patient also has Condition B }\end{array}$ & $\begin{array}{l}\text { - Screening for colon cancer may not be appropriate } \\
\text { in patients with advanced disease (e.g., metastatic } \\
\text { prostate cancer, end-stage renal disease, } \\
\text { or severe dementia) }{ }^{78}\end{array}$ \\
\hline Diagnosis & $\begin{array}{l}\text { Diagnostic uncertainty may arise if Condition } \\
\text { A and Condition B have overlapping } \\
\text { clinical manifestations }\end{array}$ & $\begin{array}{l}\text { - Diagnosing the cause of ankle swelling may } \\
\text { be challenging in a patient with heart failure and } \\
\text { arthritis }\end{array}$ \\
\hline Treatment & $\begin{array}{l}\text { Adherence to clinical practice guidelines for } \\
\text { Condition A may exacerbate Condition B }\end{array}$ & $\begin{array}{l}\text { - For a patient with osteoporosis and diabetic peripheral } \\
\text { neuropathy, clinical guidelines for osteoporosis } \\
\text { recommend weight-bearing exercise, but these } \\
\text { exercises could exacerbate the patient's peripheral } \\
\text { neuropathy }{ }^{44} \\
\text { - Cardioselective beta-blockers are recommended in a } \\
\text { patient with heart failure, but are contraindicated } \\
\text { due to the patient's asthma }\end{array}$ \\
\hline Follow-up & $\begin{array}{l}\text { Performance measures for long-term management } \\
\text { of Condition A may be inappropriate due to the } \\
\text { presence of Condition B }\end{array}$ & $\begin{array}{l}\text { For a patient with diabetes, performance measures } \\
\text { for monitoring and addressing glycemic control } \\
\text { may not be appropriate if the individual has a } \\
\text { severe illness such as stroke or end-stage } \\
\text { hepatic disease }\end{array}$ \\
\hline
\end{tabular}

*Categories represent care processes that are frequently evaluated in quality-of-care literature 79

can cause heart failure-like symptoms of fatigue and shortness of breath, respectively. ${ }^{57}$

\section{OPPORTUNITIES FOR CLINICAL PRACTICE, RESEARCH, AND POLICY}

There are a number of opportunities to improve care for multimorbid patients by incorporating the concept of comorbidity interrelatedness into clinical practice, research, and policy (Table 2).

\section{Comorbidity Interrelatedness Measures}

The concept of comorbidity interrelatedness is most likely to contribute to research and quality assessments if it can be operationalized with readily available information. An ideal measure would be one that uses data from an electronic health record (EHR) to construct overall and disease-

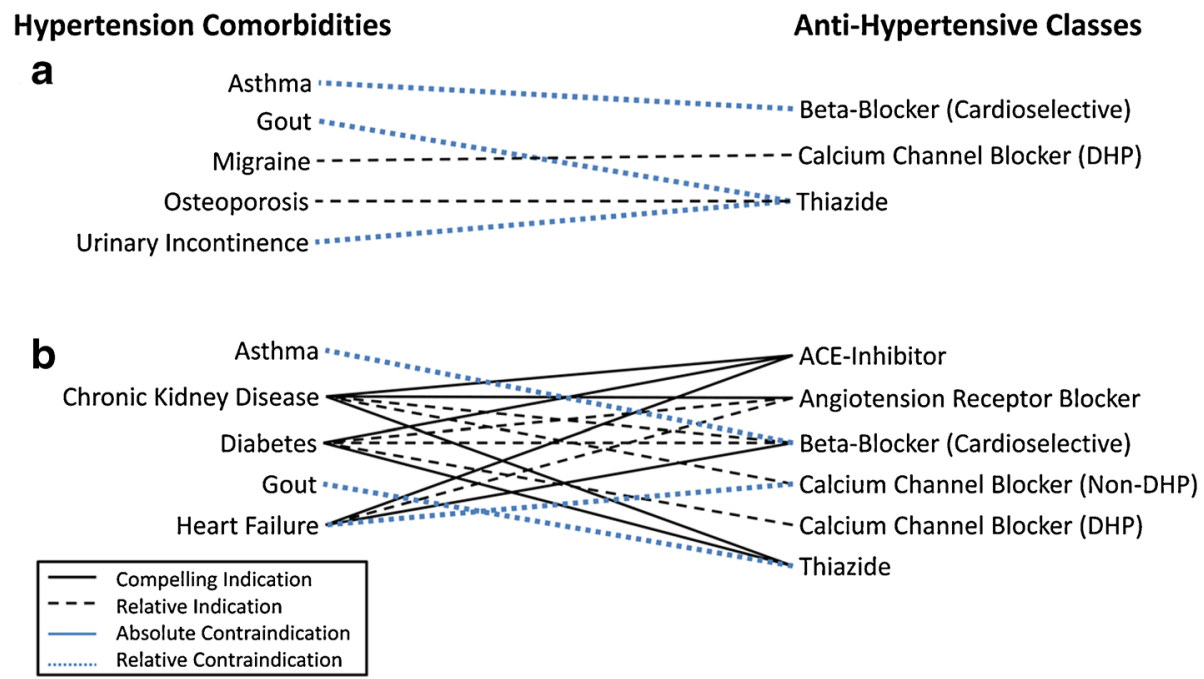

Figure 2. Variation in hypertension management complexity among two individuals with five comorbid conditions, but different levels of comorbidity interrelatedness. This figure illustrates variations in hypertension management complexity for two individuals who each have five hypertension-related comorbidities (conditions for which published clinical evidence and/or guidelines describe interactions with one or more anti-hypertensive classes). ${ }^{75-77}$ Comorbidity interrelatedness varies for Individuals A and B, who have five vs. 16 instances of potential comorbidity-medication interactions (respectively), based on indications and contraindications for one of six common anti-hypertensive classes, and one vs. three instances (respectively) in which the same drug is both indicated and contraindicated on account of their comorbid conditions. *ACE = Angiotensin Converting Enzyme; DHP = Dihydropyridine; Non-DHP = Non-Dihydropyridine. 
Table 2. Recommendations for Incorporating Comorbidity Interrelatedness into Clinical Practice, Research, and Policy

\begin{tabular}{|c|c|c|}
\hline & Recommendation & Potential impact \\
\hline $\begin{array}{l}\text { Comorbidity } \\
\text { Interrelatedness } \\
\text { Measures }\end{array}$ & $\begin{array}{l}\text { - Develop comorbidity interrelatedness measures that } \\
\text { use electronic health record data to quantify the degree } \\
\text { to which condition interactions affect clinical } \\
\text { management complexity }\end{array}$ & $\begin{array}{l}\text { - Further understanding about how combinations of } \\
\text { conditions affect clinical decisions and quality of care, } \\
\text { clinical outcomes, and health care utilization; enhance } \\
\text { value of case mix adjustment; advance clinical } \\
\text { guidelines, performance measurement, decision } \\
\text { support tools, and research about multimorbidity }\end{array}$ \\
\hline Clinical Practice Guidelines & $\begin{array}{l}\text { - Extend clinical guidelines, and where needed, } \\
\text { develop new guidelines, to promote best care } \\
\text { practices when comorbid conditions may alter } \\
\text { a patient's clinical course or ability to } \\
\text { achieve treatment benefit }\end{array}$ & $\begin{array}{l}\text { - Provide evidence-based guidance about how to } \\
\text { prioritize treatment options for patients with multiple } \\
\text { chronic conditions; encourage individualized care } \\
\text { based on a patient's specific benefit/risk profile }\end{array}$ \\
\hline $\begin{array}{l}\text { Performance Measurement } \\
\text { and Reimbursement }\end{array}$ & $\begin{array}{l}\text { - Develop performance measures that incorporate } \\
\text { a patient's comorbidity profile and the complexity } \\
\text { of the required management decisions }\end{array}$ & $\begin{array}{l}\text { - Incentivize the delivery of high-quality care for } \\
\text { multimorbid patients by rewarding cognitively } \\
\text { demanding, time-consuming, patient-centered care }\end{array}$ \\
\hline \multirow[t]{3}{*}{ Research and Clinical Care } & $\begin{array}{l}\text { - Consider how individual patients' conditions, } \\
\text { social support, environment, and goals may interact } \\
\text { in ways that affect their self-management, treatment } \\
\text { effectiveness, and overall well-being }\end{array}$ & $\begin{array}{l}\text { - Promote highly individualized care that incorporates } \\
\text { a patient's specific conditions and life circumstances }\end{array}$ \\
\hline & $\begin{array}{l}\text { - Include, and potentially oversample, patients } \\
\text { with comorbidities in clinical effectiveness studies }\end{array}$ & $\begin{array}{l}\text { - Identify differential treatment effects by comorbidity } \\
\text { and overall health status; improve representativeness } \\
\text { of clinical evidence for patients with multiple } \\
\text { chronic conditions }\end{array}$ \\
\hline & $\begin{array}{l}\text { - Include patient-reported measures as covariates } \\
\text { and outcomes in research }\end{array}$ & $\begin{array}{l}\text { - Generate evidence about quality of life and other } \\
\text { outcomes that are of importance to many patients } \\
\text { with multiple chronic conditions }\end{array}$ \\
\hline
\end{tabular}

specific comorbidity interrelatedness scores, akin to indices such as the Charlson score ${ }^{20}$ that use diagnostic codes to quantify comorbidity number and burden of disease. For example, in the case of hypertension management (described above and in Fig. 2), one might consider a score that comprises the sum of all possible comorbidity-medication interactions (based on a patient's comorbidity profile), weighted for conditions that generate co-occurring indications and contraindications for the same medications. Such a score, while rudimentary, could help illuminate the degree to which a patient's conditions affect decisions about medical management. Integrating this information with a patient's comorbidity number and severity could potentially enhance the value of case mix adjustment, and could also be employed in the advancement of clinical guidelines, performance measures, decision support tools, and research efforts that aim to improve care for patients with multiple chronic conditions.

There are several challenges that will need to be addressed when operationalizing the concept of comorbidity interrelatedness. First, given the heterogeneity of conditions and their relationships, it may be complicated to consider all relevant interactions, although this challenge may diminish as greater numbers of EHRs program clinically meaningful comorbidity-drug interactions into alert tools. Another limitation is that the quality of a comorbidity interrelatedness score relies on the strength of the evidence supporting comorbidity interactions, which may be influenced by frequent exclusion of multimorbid and older patients from clinical trials. ${ }^{58}$ In addition, harnessing EHR data to capture comorbidity interrelatedness will be subject to the limita- tions inherent to the underlying data, including outdated or inaccurate diagnostic codes and medication information, and incomplete data for patients who receive care within more than one health system. At this time, however, the EHR is the official system of record and remains the best option for developing tools that quantify patient complexity in various domains. Furthermore, as EHRs expand to include information about a broader range of patient characteristics, including social and environmental factors, it may be possible to develop increasingly sophisticated and multidimensional measures of patient complexity.

\section{Clinical Practice Guidelines}

In order for clinical guidelines to be applicable to multimorbid patients, they need to provide recommendations about best care practices when a patient has clinically relevant comorbid conditions (i.e., conditions that are likely to alter a patient's clinical course or his or her ability to achieve treatment benefit). ${ }^{28,30,59,60}$ Guidelines that incorporate comorbidity interrelatedness in this way are likely to be of particular value within the geriatrics community, where it is widely accepted that patients and providers need to synthesize and prioritize treatment options when a patient has multiple chronic conditions. ${ }^{61}$ An example of one such effort is the 2012 consensus report copublished by the American Geriatrics Society and the American Diabetes Association, which provides guidelines for treating diabetes in the context of geriatric conditions such as dementia, functional impairment, and urinary incontinence. ${ }^{62}$ 
As EHRs become increasingly pervasive, there may be opportunities to transition from disease-oriented clinical guidelines to more patient-centered guidelines that consider individuals' multiple, interacting health problems and quantify the potential risks and benefits of alternate treatment options. In addition to improving care for individual patients, this approach may be cost-effective in certain instances. For example, simulation studies have shown that basing decisions about antihypertensive or statin therapy on a patient's overall cardiovascular risk profile rather than on a single risk factor (e.g., blood pressure or cholesterol, respectively) is a costeffective approach that yields clinical benefits. ${ }^{63,64}$ There is also a need for guidelines that focus on non-disease-specific challenges that commonly arise in caring for multimorbid patients. The National Quality Forum recently outlined a number of priority areas for measurement development for patients with multiple chronic conditions, including optimizing function, facilitating transitions between providers and sites of care, avoiding inappropriate care, and shared decision-making. ${ }^{65}$

\section{Performance Measures and Reimbursement}

Just as care guidelines need to consider clinically relevant comorbid conditions, performance measures, which encourage the translation of clinical guidelines into practice, need to account for multimorbidity. While in certain circumstances it may be appropriate to simply exclude clinically complex patients from reporting requirements, ${ }^{53}$ this approach could remove incentives to provide high-quality care to complicated and vulnerable patients. ${ }^{60}$ A preferable strategy is to develop performance measures using clinical reasoning that considers multiple patient factors. For example, an advisory panel on hypertension performance measures recommended accounting for control of severe psychiatric or addictive illness, as achieving control of these conditions is frequently necessary before hypertension can be appropriately addressed. ${ }^{66}$ Building on such measures to incorporate comorbidity interrelatedness may offer an opportunity to reward especially clinically challenging and time-consuming care. ${ }^{34,53,60}$

One method for incorporating comorbidity interrelatedness into performance measurement would be to weight clinical care based on the complexity of management decisions and/or the clinical benefit to an individual patient. $^{60}$ Such an algorithm would ideally incorporate complexity arising from interactions among conditions, as well as patient preferences. This strategy would reward clinicians for the cognitive load required to provide more challenging care, as well as for addressing a complex patient's needs in the most clinically meaningful and patient-centered way. Such a strategy could augment current efforts to develop performance measures that reward highvalue care. ${ }^{67,68}$ Similar concepts, if incorporated in Evaluation and Management Coding guidelines, could potentially reimburse providers for the cognitive services required in highly complex clinical cases.

\section{Research and Clinical Care}

There is a clear need for stronger evidence to inform care practices when patients have multiple, interacting conditions. This will require that clinical effectiveness studies include, or even oversample, patients with comorbidities, and researchers examine potential differential treatment effects by comorbidity type and overall health status. ${ }^{69,70}$ There is also an opportunity to enhance clinical care for multimorbid patients by encouraging discussions about how patients' comorbidities interact and affect their self-management practices and overall well-being. For example, patients with multiple conditions may more readily accommodate a new treatment if their provider encourages them to link it to their care for a comorbid condition. ${ }^{71}$ By addressing related comorbidities as a unit or sequentially, clinicians may reduce the number of disparate clinical domains (and associated cognitive burden) that both they and their patients must consider.

While addressing comorbidity interrelatedness in clinical guidelines, performance measures, research, and clinical settings has the potential to improve care for multimorbid patients, this strategy represents only one component of a much-needed multifaceted quality improvement effort. There is growing appreciation for the importance of understanding individuals' goals, function, and quality of life, especially when a patient has complex health care needs. ${ }^{30,72,73}$ This shift away from a disease-oriented focus and toward a more patientcentered approach will require systemic changes in both health care delivery practices and medical education. Meanwhile, there is increasing demand for researchers to use validated patient-reported measures, ${ }^{74}$ to ensure that studies reflect outcomes of importance to multimorbid patients.

\section{CONCLUSION}

In conclusion, multimorbidity is likely to continue generating complex challenges for clinical care well into the future. Incorporating the concept of comorbidity interrelatedness into clinical and research efforts is likely to enhance the care we provide for patients with multiple chronic conditions. While it is daunting to consider how to best operationalize the ways in which different conditions interact and affect a patient's health and health care needs, pursuing simple solutions under such circumstances may be unwise. Only by embracing and exploring the complex dimensions of multimorbidity are we likely to develop guidelines and practices that optimize care for patients with multiple chronic conditions. 


\section{Acknowledgements:}

Contributors: The authors thank Danielle Cohen, Emily Jenchura, and Cindie Slightam for their assistance with literature reviews, and Harold Sox, MD, for his thoughtful review of the manuscript. Views expressed are those of the authors and not necessarily those of the Department of Veterans Affairs.

Funders: This study was supported in part by Department of Veterans Affairs Health Services Research and Development (HSR\&D) grant IMV 04-062 (PI: Goldstein). Dr. Zulman is supported by a HSR\&D Career Development Award (CDA 12-173).

Prior Presentations: This work was presented at the Society for General Internal Medicine Annual Meeting in May, 2012.

Conflict of Interest: The authors declare that they do not have a conflict of interest.

Corresponding Author: Donna M. Zulman, MD, MS; Center for Health Care Evaluation, VA Palo Alto Health Care System, Division of General Medical Disciplines, Stanford University, 795 Willow Road (152- MPD), Menlo Park, CA 94025, USA (e-mail:dzulman@stanford.edu).

\section{REFERENCES}

1. Anderson G. Chronic care: Making the case for ongoing care. 2010; http://www.rwjf.org/content/dam/web-assets/2010/01/chronic-care. Accessed 11/28/12.

2. Lee TA, Shields AE, Vogeli C, et al. Mortality rate in veterans with multiple chronic conditions. J Gen Intern Med. Dec 2007;22(Supp 3):403-407.

3. Fortin M, Bravo G, Hudon C, et al. Relationship between multimorbidity and health-related quality of life of patients in primary care. Qual Res: Int $J$ Qual Life Asp Treat Care Rehabil. Feb 2006;15(1):83-91.

4. Verbrugge LM, Lepkowski JM, Imanaka Y. Comorbidity and its impact on disability. Milbank Q. 1989;67(3-4):450-484.

5. Alecxih L, Shen S, Chan I, Taylor D, Drabek J, Group TL. Individuals Living in the Community with Chronic Conditions and Functional Limitations: A Closer Look. Prepared for the Office of the Assistant Secretary for Planning \& Evaluation, U.S. Department of Health and Human ServicesJanuary, 2010.

6. Schneider KM, O'Donnell BE, Dean D. Prevalence of multiple chronic conditions in the United States' Medicare population. Health Qual Life Outcomes. 2009;7:82.

7. Wolff JL, Starfield B, Anderson G. Prevalence, expenditures, and complications of multiple chronic conditions in the elderly. Arch Intern Med. 2002;162(20):2269-2276.

8. Lehnert T, Heider D, Leicht $\mathbf{H}$, et al. Review: Health care utilization and costs of elderly persons with multiple chronic conditions. Med Care Res Rev. Aug 2011;68(4):387-420.

9. Centers for Medicare and Medicaid Services. Chronic Conditions Among Medicare Beneficiaries, Chartbook, 2012 Edition. Baltimore, MD. 2012.

10. Safford MM, Allison JJ, Kiefe CI. Patient complexity: More than comorbidity. The vector model of complexity. J Gen Intern Med. 2007;22(Suppl 3):382-390.

11. Boyd CM, Ritchie CS, Tipton EF, Studenski SA, Wieland D. From bedside to bench: summary from the American Geriatrics Society/ National Institute on Aging research conference on comorbidity and Multiple Morbidity in older adults. Aging Clin Exp Res. Jun 2008;20(3):181-188.

12. Valderas JM, Starfield B, Sibbald B, Salisbury C, Roland M. Defining comorbidity: implications for understanding health services research. Ann Fam Med. 2009;7:357-363.

13. van den Akker M, Buntinx F, Knottnerus JA. Comorbidity or multimorbidity: What's in a name? A review of literature. Eur J Gen Pract. 1996;2(2):65-70.
14. Fortin M, Soubhi H, Hudon C, Bayliss EA, van den Akker M. Multimorbidity's many challenges. BMJ. 2007;334(7602):1016-1017.

15. Boyd C, Fortin M. Future of multimorbidity research: How should understanding of multimorbidity inform health system design? Public Health Rev. 2010;32(2):451-474.

16. Starfield L, Bernhardt. Comorbidity: implications for the importance of primary care in case management. Ann Fam Med. 2003;1:8-14.

17. Fultz NH, Ofstedal MB, Herzog AR, Wallace RB. Additive and interactive effects of comorbid physical and mental conditions on functional health. J Aging Health. Aug 2003;15(3):465-481.

18. de Groot V, Beckerman H, Lankhorst GJ, Bouter LM. How to measure comorbidity. a critical review of available methods. J Clin Epidemiol. 2003;56(3):221-229.

19. Linn BS, Linn MW, Gurel L. Cumulative illness rating scale. J Am Geriatr Soc. May 1968;16(5):622-626.

20. Charlson ME, Pompei P, Ales KL, Mackenzie CR. A new method of classifying prognostic comorbidity in longitudinal studies: development and validation. J Chronic Dis. 1987;40(5):373-383.

21. Deyo RA, Cherkin DC, Ciol MA. Adapting a clinical comorbidity index for use with ICD-9-CM administrative databases. J Clin Epidemiol. 1992;45(6):613-619.

22. Imamura $\mathbf{K}$, McKinnon $\mathbf{M}$, Middleton $\mathbf{R}$, Black N. Reliability of a comorbidity measure: the index of co-existent disease (ICED). J Clin Epidemiol. 1997;50(9):1011-1016.

23. Malenka DJ, McLerran D, Roos N, Fisher ES, Wennberg JE. Using administrative data to describe casemix: a comparison with the medical record. J Clin Epidemiol. 1994;47(9):1027-1032.

24. Piette JD, Kerr EA. The impact of comorbid chronic conditions on diabetes care. Diabetes Care. Mar 2006;29(3):725-731.

25. Mercer SW, Gunn J, Bower P, Wyke S, Guthrie B. Managing patients with mental and physical multimorbidity: changes are needed in policy, research, and practice. BMJ (Clinical research ed.). 2012;345(e5559).

26. Yoon J, Zulman DM, Scott JY, Maciejewski ML. Costs associated with multimorbidity among VA patients. Medical Care. [In Press].

27. Steinman MA, Lee SJ, Boscardin WJ, et al. Patterns of multimorbidity in elderly veterans. J Am Geriatr Soc. 2012;60(1872-1880).

28. Boyd CM, Leff B, Wolff $\mathbf{J L}$, et al. Informing clinical practice guideline development and implementation: prevalence of coexisting conditions among adults with coronary heart disease. J Am Geriatr Soc. May 2011;59(5):797-805.

29. Nardi R, Scanelli G, Corrao S, Iori I, Mathieu G, Cataldi AR. Comorbidity does not reflect complexity in internal medicine patients. Eur $\mathrm{J}$ Intern Med. 2007;18(5):359-368.

30. Guiding Principles for the Care of Older Adults with Multimorbidity: An Approach for Clinicians: American Geriatrics Society Expert Panel on the Care of Older Adults with Multimorbidity. J Am Geriatr Soc. Sep 192012.

31. Krein SL, Hofer TP, Holleman R, Piette JD, Klamerus ML, Kerr EA. More than a pain in the neck: How discussing chronic pain affects hypertension medication intensification. J Gen Intern Med. Aug 2009;24(8):911-916.

32. Higashi T, Wenger NS, Adams JL, et al. Relationship between number of medical conditions and quality of care. N Engl J Med. 2007;356(24):2496-2504.

33. Petersen LA, Woodard LD, Henderson LM, Urech TH, Pietz K. Will hypertension performance measures used for pay-for-performance programs penalize those who care for medically complex patients? Circulation. 2009;119(23):2978-2985.

34. Halanych JH, Safford MM, Keys WC, et al. Burden of comorbid medical conditions and quality of diabetes care. Diabetes Care. Dec 2007;30(12):2999-3004.

35. Min LC, Reuben DB, MacLean CH, et al. Predictors of overall quality of care provided to vulnerable older people. J Am Geriatr Soc. Oct 2005;53(10):1705-1711.

36. Turner BJ, Hollenbeak CS, Weiner M, Ten Have T, Tang SS. Effect of unrelated comorbid conditions on hypertension management. Ann Intern Med. 2008;148(8):578-586.

37. Redelmeier DA, Tan SH, Booth GL. The treatment of unrelated disorders in patients with chronic medical diseases. N Engl J Med. 1998;338(21):1516-1520.

38. Kerr EA, Zikmund-Fisher BJ, Klamerus ML, Subramanian U, Hogan MM, Hofer TP. The role of clinical uncertainty in treatment decisions for diabetic patients with uncontrolled blood pressure. Ann Intern Med. 2008; 148(10):717-727.

39. Frayne SM, Halanych JH, Miller DR, et al. Disparities in diabetes care: impact of mental illness. Arch Intern Med. 2005;165(22):2631-2638. 
40. Braunstein JB, Anderson GF, Gerstenblith G, et al. Noncardiac comorbidity increases preventable hospitalizations and mortality among Medicare beneficiaries with chronic heart failure. J Am Coll Cardiol. 2003;42(7): 1226-1233.

41. Krein SL, Bingham CR, McCarthy JF, Mitchinson A, Payes J, Valenstein M. Diabetes treatment among VA patients with comorbid serious mental illness. Psychiatr Serv. Jul 2006;57(7):1016-1021.

42. Kerr EA, Heisler M, Krein SL, et al. Beyond comorbidity counts: How do comorbidity type and severity influence diabetes patients' treatment priorities and self-management? J Gen Intern Med. Dec 2007;22(12):16351640.

43. Zulman DM, Kerr EA, Hofer TP, Heisler M, Zikmund-Fisher BJ. Patient-provider concordance in the prioritization of health conditions among hypertensive diabetes patients. J Gen Intern Med. May 2010;25(5):408-414

44. Boyd CM, Darer J, Boult C, Fried LP, Boult L, Wu AW. Clinical practice guidelines and quality of care for older patients with multiple comorbid diseases: implications for pay for performance. JAMA. 2005;294(6):716-724.

45. Simon H. The architecture of complexity. Proc Am Philos Soc. 1962;106(6):467-482.

46. Wood RE. Task complexity: definition of the construct. Organ Behav Hum Decis Process. 1986;37(1):60-82.

47. Sintchenko V, Coiera E. Decision Complexity Affects the Extent and Type of Decision Support Use. AMIA Annu Symp Proc. 2006:725-728.

48. Kannampallil TG, Schauer GF, Cohen T, Patel VL. Considering complexity in healthcare systems. J Biomed Inform. 2011;44(6):943-947.

49. Malhotra S, Jordan D, Shortliffe E, Patel VL. Workflow modeling in critical care: piecing together your own puzzle. J Biomed Inform. 2007;40(2):81-92.

50. Cohen T, Blatter B, Almeida C, Shortliffe E, Patel V. A cognitive blueprint of collaboration in context: distributed cognition in the psychiatric emergency department. Artif Intell Med. 2006;37(2):73-83.

51. Tinetti ME, Bogardus ST, Agostini JV. Potential pitfalls of diseasespecific guidelines for patients with multiple conditions. N Engl J Med. 2004;351(27):2870-2874.

52. Ritchie C, Kvale E, Fisch MJ. Multimorbidity: an issue of growing importance for oncologists. J Oncol Pract. 2011;7(6):371-374.

53. Pogach LM, Tiwari A, Maney M, Rajan M, Miller DR, Aron D. Should mitigating comorbidities be considered in assessing healthcare plan performance in achieving optimal glycemic control? Am J Manag Care. Mar 2007;13(3): 133-140.

54. Fried TR, Tinetti ME, Iannone L. Primary Care Clinicians' Experiences With Treatment Decision Making for Older Persons With Multiple Conditions. Arch Intern Med. 2011;171(1):75-80.

55. Bower P, Macdonald W, Harkness E, et al. Multimorbidity, service organization and clinical decision making in primary care: a qualitative study. Fam Pract. 2011;28(5):579-587.

56. Luijks HD, Loeffen MJ, Lagro-Janssen AL, van Weel C, Lucassen PL, Schermer TR. GPs' considerations in multimorbidity management: a qualitative study. Br J Gen Pract. 2012;62(600):e503-e510.

57. Dickson Vv, Buck H, Riegel B. A qualitative meta-analysis of heart failure self-care practices among individuals with multiple comorbid conditions. J Card Fail. May 2011;17(5):413-419.

58. Jadad AR, To MJ, Emara M, Jones J. Consideration of multiple chronic diseases in randomized controlled trials. JAMA. 2011;306(24):2670-2672.

59. Braithwaite RS, Concato J, Chang CC, Roberts MS, Justice AC. A framework for tailoring clinical guidelines to comorbidity at the point of care. Arch Intern Med. 2007;167(21):2361-2365.
60. Werner RM, Greenfield S, Fung C, Turner BJ. Measuring quality of care in patients with multiple clinical conditions: summary of a conference conducted by the Society of General Internal Medicine. J Gen Intern Med. Aug 2007;22(8):1206-1211.

61. Brown AF, Mangione CM, Saliba D, Sarkisian CA. Guidelines for improving the care of the older person with diabetes mellitus. J Am Geriatr Soc. May 2003;51(5 Suppl Guidelines):S265-280.

62. Kirkman M, Briscoe V, Clark N, et al. Diabetes in Older Adults: a Consensus Report. JAGS. 2012;60(12).

63. Hayward RA, Krumholz HM, Zulman DM, Timbie J, Vijan S. Optimizing statin treatment for primary prevention of coronary artery disease. Ann Intern Med. 2010;152:69-77.

64. Eddy D, Adler J, Patterson B, Lucas D, Smith K, Morris RL. Individualized guidelines: the potential for increasing quality and reducing costs. Ann Intern Med. 2011;154:627-634.

65. National Quality Forum. Multiple Chronic Conditions Measurement Framework. National Quality Forum; Washington, D.C.2012

66. Steinman MA, Goldstein MK. When tight blood pressure control is not for everyone: a model for performance measurement in hypertension. Joint Comm J Qual Patient Saf / Joint Comm Resour. 2010;36(4): 164-172.

67. VanLare J, Blum J, Conway P. Linking performance with payment: implementing the physician value-based payment modifier. JAMA. 2012.

68. Fisher ES, Shortell SM. Accountable care organizations: accountable for What, to Whom, and How. JAMA. 2010;304(15):1715-1716.

69. Zulman DM, Sussman JB, Chen X, Cigolle CT, Blaum CS, Hayward RA. Examining the evidence: a systematic review of the inclusion and analysis of older adults in randomized controlled trials. J Gen Intern Med. Jul 2011;26(7):783-790.

70. Boyd CM, Vollenweider D, Puhan MA. Informing Evidence-Based Decision-Making for Patients with Comorbidity: availability of necessary information in clinical trials for chronic diseases. PLoS One. 2012;7(8).

71. Morris RL, Sanders C, Kennedy AP, Rogers A. Shifting priorities in multimorbidity: a longitudinal qualitative study of patient's prioritization of multiple conditions. Chronic Illn. 2011;7(2).

72. Tinetti ME, Fried TR, Boyd CM. Designing health care for the most common chronic condition-multimorbidity. JAMA. 2012;307(23):24932494.

73. Smith S, Soubhi H, Fortin M, Hudon C, O'Dowd T. Interventions for improving outcomes in patients with multimorbidity in primary care and community settings. Cochrane Database Syst Rev. 2012(1469-493X).

74. National Institutes of Health. Patient Reported Outcomes Measurement Information System (PROMIS). 2012; http://nihpromis.org/. Accessed $10 / 4 / 12$.

75. The Management of Hypertension in the Primary Care Setting Working Group. VA/DoD Clinical Practice Guideline for Diagnosis and Management of Hypertension in the Primary Care Setting. 2004; http:// www.healthquality.va.gov/hypertension/htn04_pdf1.pdf.

76. Department of Health and Human Services. The Seventh Report of the Joint National Committee on Prevention, Detection, Evaluation, and Treatment of High Blood Pressure. 2004;4(5230).

77. Goodman LS, Hardman JG, Gilman AG, Limbird LE. Goodman and Gilman's The Pharmacological Basis of Therapeutics. New York: McGraw-Hill, Medical Division; 2001.

78. Walter LC, Davidowitz NP, Heineken PA, Covinsky KE. Pitfalls of converting practice guidelines into quality measures. Lessons learned from a VA performance measure. JAMA. 2004;291(20):2466-2470.

79. McGlynn EA, Asch SM, Adams J, et al. The quality of health care delivered to adults in the United States. N Engl J Med. 2003;348(26):2635-2645. 